\title{
Clinicopathological Study of Medullary Breast Carcinoma: An Instututional Study
}

\author{
B. R. Vani* Netra. M. Sajjan, Deepak Kumar B, Srinivasa Murthy V.
}

Dept of Pathology, ESI Medical college \&Post Graduate Institute of Medical Science and Research, Rajajinagar, Bangalore (India)

\begin{abstract}
Background: Medullary breast carcinoma (MBC) is a rare distinct type of invasive breast carcinoma with incidence being less than $5 \%$. It usually occurs under the age of 50 years, often mistaken clinically for fibroadenoma. They are divided into typical, and atypical MBC with a prerequisite of syncytial growth pattern in more than $75 \%$ of the tumor cells. MBCs are often hormone (ER, PR, Her $2 /$ neu) negative with grade 3 Nottingham's criteria but havebetter prognosis.

Methods: Retrospective study of invasive carcinoma of the breast was undertaken in Pathology department from 2009-2016. Total numbers of invasive breast carcinomas were 388, of which MBC were 12 cases histologically.Clinical detailsand gross features were noted. Histopathology and Immunohistochemistry (ER, PR, Her2/neu) slides were reviewed.

Result: Age range was 35 to 64 years, with(mean 49.08). All presented clinically as solitary lump in breast. No side predilection. Most common site affected was upper inner quadrant consitituing around 50\%(6 out of 12) of cases. Overall tumour size ranged from 2.5 to 6 cms with a mean of $3.6 \mathrm{cms}$ in typical and $4.25 \mathrm{cms}$ in atypical MBC. Grossly majority of the tumors were soft to firm in consistency. Histologically 10 out of 12 cases ( $83.3 \%$ ) were typical MBC and 2 cases were atypical MBC(16.7\%).IHC showed triple negativity in $75 \%$ of MBC with majority in typical MBC $(80 \%)$

Conclusion: $\mathrm{MBC}$ is unique type of breast carcinoma with good prognosis. Histopathology plays important role in diagnosing variants of MBC since the treatment differs.
\end{abstract}

Keywords: Medullary, Breast Carcinoma,Typical, Atypical, Histopathology, Immunohistochemistry

\section{Introduction}

Medullary breast carcinoma $(\mathrm{MBC})$ is a rare, distinct type of invasive breast carcinoma ${ }^{1}$ with incidence of less than $5 \%$ of invasive breast carcinoma. ${ }^{1,2}$ It uusually occurs under age of 50 years, often mistaken clinically for fibroadenoma. ${ }^{3} \mathrm{MBC}$ are divided into typical and atypical medullary carcinomas, with a prerequisite of syncytial growth pattern in $>75 \%$ of the tumor cells in both. Syncytial growth pattern is characterized by tumor cells arranged in sheets, usually more than four or five cells thick, separated by small amounts of loose connective tissue. ${ }^{4}$ Typical MBC is characterised by a constellation of five histological features, as defined first by Ridolfi et al in $1977 .{ }^{2}$ These carcinomas are often hormone (ER, PR, Her2/neu) negative with grade 3 Nottinghams criteria ,basal phenotype; but have a better prognosis. ${ }^{2}$ Strong association of marked inflammation with better prognosis is seen in triple negative carcinomas. ${ }^{5}$ Presence of lymphocytes and plasma cells keeps check on MBC and prevents its growth and spread. ${ }^{1}$ Overall five year survival rate is $95 \%$ in typical, $80 \%$ in atypical medullary carcinoma and $70 \%$ in invasive breast carcinoma ${ }^{6}$.
With this background, the current study was undertaken to evaluate the prevalence of $\mathrm{MBC}$ and to study the clinicopathological and immunohistochemical profile in typical and atypical MBC.

\section{Materials and Methods}

Retrospective study of invasive carcinoma of the breast was undertaken in the department of Pathology from 2009-2016. Total number of invasive breast carcinomas were 388 , of which 12 cases were MBC on histomorphology. All the 12 cases of MBCs were taken up for the study. The clinical data such as age of patient and type of surgery were collected from the medical records. Gross features of specimen and appearance on cut surface were noted. Histopathology and IHC (ER, PR, Her2/neu) slides were retrieved. Blocks were collected when slides were not available, subsequently sections were made and histopathology slides evaluated. Primary histologic feature defining both typical and atypical MBC, was presence of more than $75 \%$ syncytial tumour growth pattern and lymphoplasmacytic infiltrate in varying proportions. 
Histological features characterising typical medullary carcinoma was the constellation of five histolological features such as more than $75 \%$ syncytial growth pattern, complete lesional circumscription, moderate to marked diffuse lymphoplasmacytic infiltrate, moderate to severe nuclear pleomorphism, and lack of intraductal component and tubular differentiation. ${ }^{4}$

Atypical medullary carcinoma was defined histologically by not more than two of the following four atypical features such as margins with focal or prominent infiltrative pattern, mononuclear infiltrate mild or at the tumor margins only, benign appearing nuclei and presence of microglandular features. $^{4}$

Immunohistochemical stained slides of ER, PR, Her2/ neu were reviewed and analysed. Interpretation of ER, PR was done depending on the extent and intensity of nuclear staining as per Allred score. Interpretation of Her2/neu was done based on intensity of membrane staining pattern.

Data was analysed and evaluated using descriptive statistics such as median, range standard deviation.

\section{Result}

During the study period a total of 388 cases of invasive breast carcinoma were diagnosed out of which $12(3.1 \%)$ were MBC. Age range was 35-64 years(mean 49.08). All cases clinically presented as solitary lump in breast. There was no side predilection however the most common quadrant affected was upper inner quadrant consitituing around $50 \%$ (6 of 12) of cases. Modified radical mastectomy
(MRM) was performed in all cases since FNA was reported as carcinoma breast.

Clinical features of typical and atypical medullary carcinomas are summarized in table 1. Overall tumour size ranged from 2.5 to $6 \mathrm{~cm}$ (mean $3.70 \mathrm{~cm}$ ). Average size in typical $\mathrm{MBC}$ was $3.6 \mathrm{cms}$ (range 2.5 to $6 \mathrm{cms}$ ) and atypical MBC was $4.25 \mathrm{cms}$ (range 3.5-5.0 cms). One of the twelve cases clinically presented with involvement of nipple areola region.

Grossly majority of the tumors were soft to firm in consistency. Fine needle aspiration cytology showed large, pleomorphic, singly scattered and disintegrated tumor cells with dense lymphoplasmacytic infiltrate in the background.

Histologically 10 out of 12 cases (83.3\%) were diagnosed as typical $\mathrm{MBC}$ and 2 cases were atypical MBC (16.7\%). Histological features of typical and atypical MBCs are summarised in table 2 and3. Axillary lymph node dissection was done and lymph nodes were isolated. Overall $40 \%$ of typical MBC and $50 \%$ of atypical MBC showed metastatic deposits. (Table 2 \& 3)

IHC showed triple negativity in $75 \%$ cases of $\mathrm{MBC}$ comprising of $80 \%$ in typical and $50 \%$ in atypical $\mathrm{MBC}$

\section{Discussion}

MBC was first described by Moore and Foote in 1949.7 These are well circumscribed lesions, often soft, fleshy and tend to bulge above the surrounding parenchyma, hence the term encephaloid. ${ }^{8}$ Medullary carcinoma is

Table1: Clinical and Gross Features of Typical and Atypicalmedullary Carcinoma

\begin{tabular}{|l|c|c|c|c|c|}
\hline & Age & Type of Surgery & Side Quad & Size (cm) & Nipple/Areola \\
\hline Case 1 Typical MBC & 35 & MRM & Right UOQ & $3 \times 2.4 \times 2$ & No \\
\hline Case 2 Typical MBC & 58 & MRM & Right UIQ & $3 \times 2 \times 2$ & No \\
\hline Case-3 Typical MBC & 64 & MRM & Right central Q & $4 \times 3 \times 2$ & ulceration noted \\
\hline Case 4 Typical MBC & 47 & MRM & Left LOQ & $3 \times 2.5 \times 2$ & No \\
\hline Case 5 Typical MBC & 40 & MRM & Left LOQ & $6 \times 5 \times 3$ & No \\
\hline Case 6 Typical MBC & 45 & MRM & Right UIQ & $4 \times 3 \times 3$ & No \\
\hline Case 7 Typical MBC & 42 & MRM & Left UIQ & $4 \times 3 \times 2.5$ & No \\
\hline Case 8 Typical MBC & 45 & Biopsy & Left UIQ & $3 \times 2 \times 2.6$ & No \\
\hline Case 9 Typical MBC & 45 & MRM & Right UOQ & $3.5 \times 2.5 \times 2$ & No \\
\hline Case 10 Typical MBC & 50 & MRM & Right UIQ & $2.5 \times 2.1 \times 2$ & No \\
\hline Case 11 Atypical MBC & 60 & MRM & Left UIQ & $5 \times 4 \times 3$ & No \\
\hline Case 12 Atypical MBC & 58 & MRM & Left UOQ & $3.5 \times 3 \times 3$ & \\
\hline
\end{tabular}

UIQ-Upper inner quadrant, LOQ-Lower outer quadrant, UOQ-Upper outer quadrant, ,RCQ-Right central quadrant 
Table2: Histopathological Features of Typical Medullary Carcinoma

\begin{tabular}{|c|c|c|c|c|c|c|c|c|c|c|}
\hline & Case 1 & Case 2 & Case3 & Case4 & Case 5 & Case 6 & Case 7 & Case 8 & Case 9 & Case 10 \\
\hline $\begin{array}{l}>75 \% \text { Syncyial } \\
\text { group }\end{array}$ & + & + & + & + & + & + & + & + & + & + \\
\hline Lp infiltrate & marked & marked & Marked & mod & mod & marked & mod & marked & marked & mod \\
\hline pleomorphism & mod & mod & Marked & mod & marked & marked & marked & marked & marked & mod \\
\hline IDC features & - & - & - & - & - & - & - & - & - & - \\
\hline $\begin{array}{l}\text { Necrosis \% } \\
1-10 \% \\
>10 \%\end{array}$ & nil & nil & $1-10 \%$ & nil & nil & nil & nil & nil & nil & nil \\
\hline Tumor border & wc & wc & wc & Push & push & wc & wc & wC & wc & push \\
\hline Lymph node & 0 & $1 / 6$ & $0 / 16$ & 0 & $0 / 7$ & $0 / 3$ & $1 / 6$ & $1 / 4$ & $0 / 6$ & $2 / 8$ \\
\hline SBR Grade & II & II & III & II & III & III & III & III & III & II \\
\hline Stage & Ila & Illb & IIIb & Ila & Ilb & Ila & Ilb & Ilb & Ila & Ilb \\
\hline $\begin{array}{l}\text { ER/PR/HER } 2 \\
\text { neu }\end{array}$ & $-/-/-$ & $+/+/-$ & $+/+/+$ & $-/-/-$ & $-/-/-$ & $-/-/-$ & $-/-/-$ & $-/-/-$ & $-/-/-$ & $-/-/-$ \\
\hline
\end{tabular}

Table 3 : Histopathological Features of Atypical Medullary Carcinoma

\begin{tabular}{|l|c|c|}
\hline & Case11 & Case 12 \\
\hline$>75 \%$ syncytial group & mild & mild \\
\hline Lp infiltrate & marked & mod \\
\hline pleomorphism & DCIS CIS \\
\hline DCIS/IDC & & LVI \\
\hline $\begin{array}{l}\text { Necrosis } \% \\
>10 \%\end{array}$ & $>10 \%$ & nil \\
\hline Tumor border & & infiltr \\
\hline Lymph node & $3 / 8$ & infiltr \\
\hline SBR Grade & II & $12 / 26$ \\
\hline Stage & Ila & II \\
\hline ER/PR/HER 2 neu & II-/- & IIIC \\
\hline
\end{tabular}

Lp: Lymphoplasmacytic infiltrate; DCIS/IDC: Ductal carcinoma in situ/ Invasive ductal carcinoma; SBR : Modified scarff bloom richardson grading; mod: moderate; wc: well circumscribed; push: pushing borders; infiltr: infiltrating margins

Annals of Pathology and Laboratory Medicine, Vol. 5, Issue 1, January, 2018 


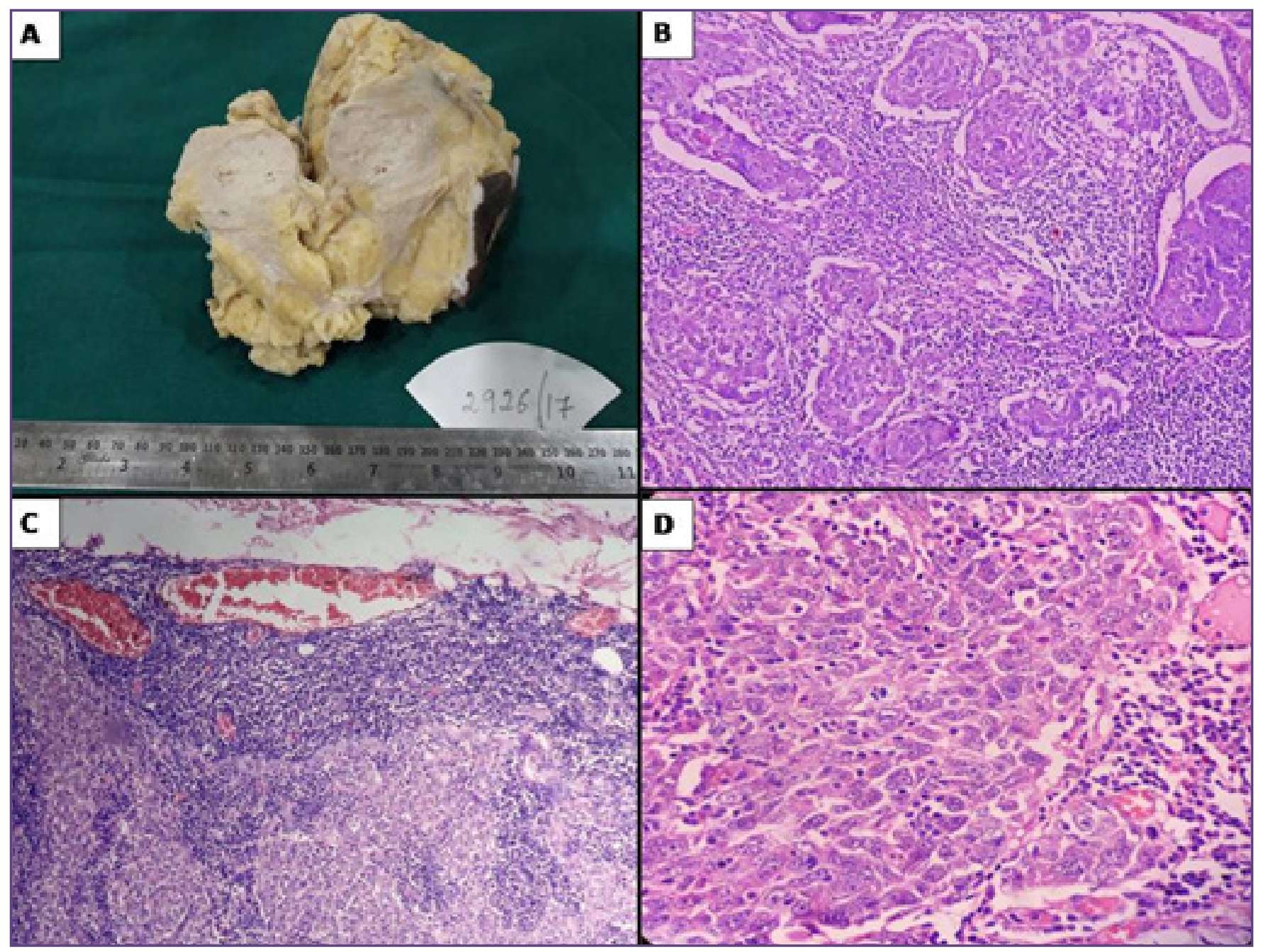

Fig. 1: Typical MBC: A - GROSS showing well circumscribed, nodular fleshy grayish pink lesion; B -Syncytial growth pattern of tumor cells (H\&E 10x); C- Circumscribed tumor with moderate to marked lymphoplasmacytic infiltrate(H\&E 4x); D -Cells exhibiting high grade nuclear features (H\&E $40 \mathrm{x}$ )

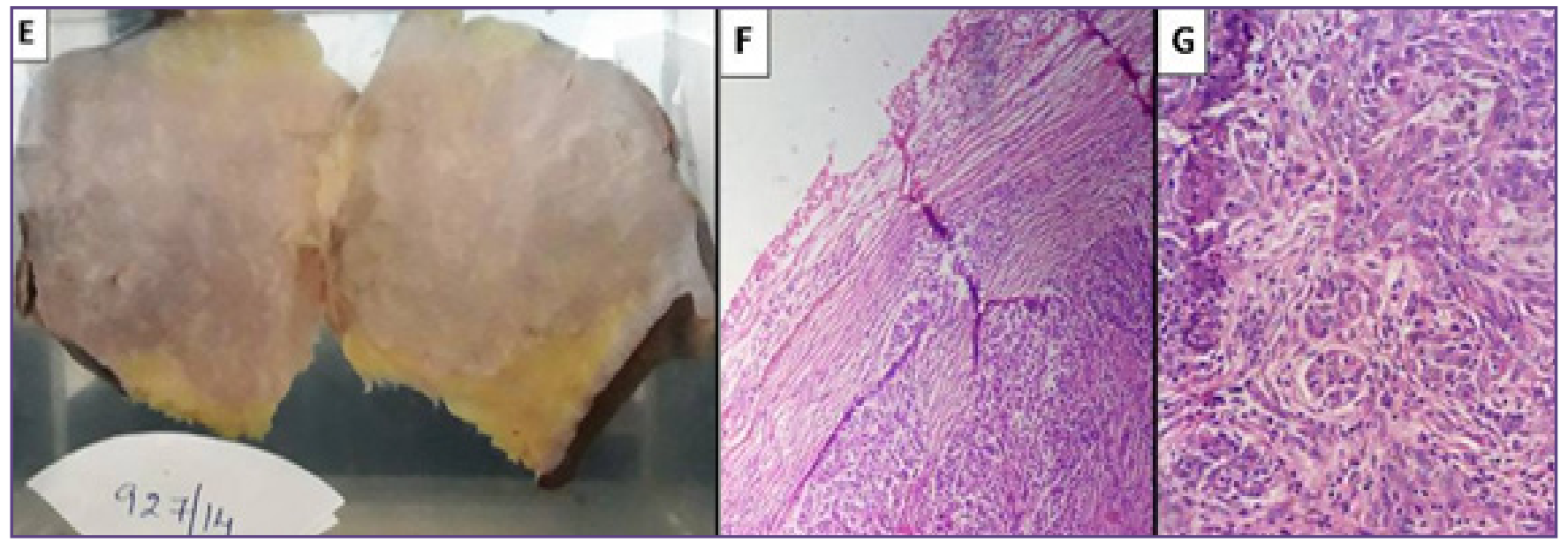

Fig. 2: ATYPICAL MBC: E- Gross showing Large fleshy grey pink with infiltrative margins.; F - Tumor exhibiting infiltrative margins (H\&E 10x); G - High power showing glandular pattern (H\&E 40x) 


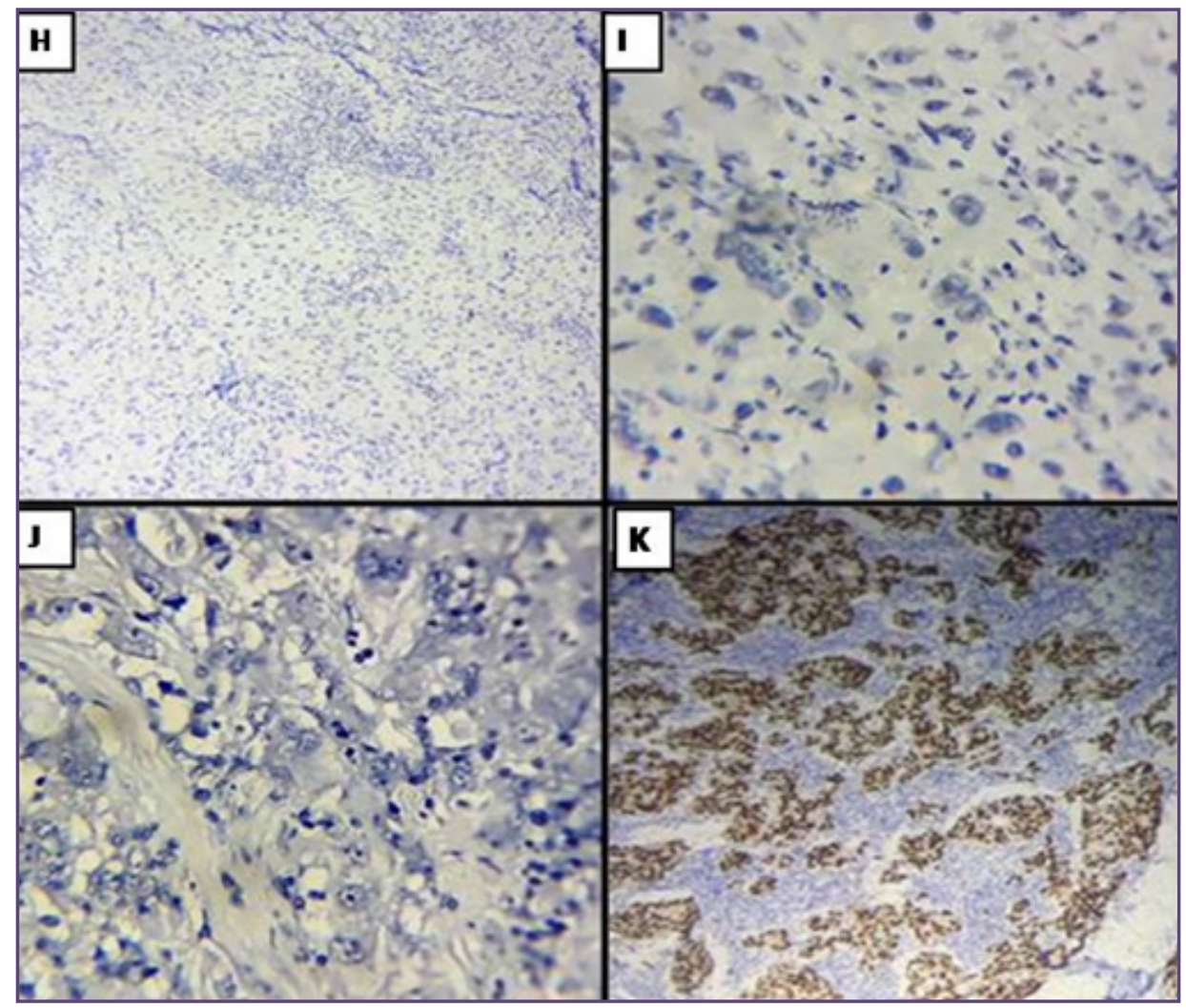

Fig. 3: Immunohistochemistry showing triple negativity (ER, PR, Her2neu negative) in fig $\mathrm{H}, \mathrm{I}, \mathrm{J}$ respectively and fig $\mathrm{K}$ showing ER positivity in one of the typical MBC.

an uncommon tumor accounting for less than $5 \%$ of all invasive breast carcinomas. Prevalence of $\mathrm{MBC}$ in our study was $3.1 \%$ which is in concordance with various studies available in literature. ${ }^{1,2}$ Radiologically and clinically MBC mimics fibroadenoma due to its smaller size. Median size of the tumor ranged from 2-3 $\mathrm{cms}^{3}$ Clinically they present as circumscribed tumor with tendency for cystic degeneration and ulceration of the skin in few. In present study 1 out of 12 cases showed ulceration of the skin involving the nipple areola region. According to the study conducted by Ridolfi et $\mathrm{al}^{9}$, age of presentation ranged from 47-52 years. Our study showed similar age range. $60 \%$ of typical MBC occurred in age group of 40-49 years, while $50 \%$ of atypical MBC occurred in age group of 50-59 and 60-69 years (Table 1).

Grossly, medullary carcinomas are circumscribed, nodular, fleshy with grey pink appearance on cut surface (Fig 1A). Cystic degeneration, hemorrhage and necrosis are seen. Histologically, MBC is characterized by syncytial growth pattern (Fig 1B), complete circumscription (Fig1C), moderate to marked diffuse lymphoplasmacytic infiltrate (Fig 1C), moderate to severe nuclear pleomorphism(Fig 1D) and absence of glandular pattern. Presence ofmicroglandular pattern, infiltrative margins, mild mononuclear infiltrate and benign appearing nuclei characterizes atypical $\mathrm{MBC}^{4}$. Atypical MBC is synonymously named by some authors as invasive carcinoma with medullary like features. ${ }^{10}$

Wargotz et al reported 5 year survival rate of $95 \%, 80 \%$ and $70 \%$ in typical $\mathrm{MBC}$, atypical $\mathrm{MBC}$ and invasive breast carcinomas respectively. In our study atypical MBC distinctly had IDC like areas with glandular formation of tumour cells and infiltrative tumour margins (Figure 2 F, G). The incidence of atypical MBC was $16.7 \%$, similar to study conducted by Jagtap et al. ${ }^{1}$ Histopathological categorization of atypical $\mathrm{MBC}$ is necessary since adjuvant chemotherapy is given along with MRM, unlike in typical medullary carcinoma where MRM is the mainstay of treatment ${ }^{10}$. The average number of lymph nodes found grossly in axillary dissection specimen from a patient with $\mathrm{MBC}$ is greater than for the other types of carcinoma. This difference is due to the greater ease of finding enlarged hyperplastic reactive lymph nodes in $\mathrm{MBC} .{ }^{11}$ Most studies indicate that the incidence of axillary lymph node metastases is lower in patients with medullary carcinomas $(19 \%$ - 
$46 \%$ ) than in those with atypical medullary carcinomas (30\%-52\%) or invasive ductal carcinomas $(29 \%-65 \%) .^{12}$ In our study, the number of positive metastatic lymph node involvement were more in atypical MBC (50\%) compared to typical MBC. Typically MBCs are almost invariably triple hormone receptor negative, although some typical and atypical MBCs are ER, PR and/or HER-2 positive, indicating the heterogeneity of this type of breast carcinomas. ${ }^{13}$ In our study IHC showed triple negativity in $75 \%$ cases of MBC (Fig 3) with majority of in typical MBC (80\%) similar to study conducted by Jagtap et $\mathrm{al}^{1}$.Of the two cases of atypical MBC, one case was ER, PR positive, while Her 2/neu negativity was noted in both.

Typical MBC and atypical MBC are similar in presentation, treatment and prognosis. ${ }^{14,15} \mathrm{Few}$ cases of atypical MBCs were treated with adjuvant chemotherapy based on Her2 status and lymph node status along with Modified radical mastectomy in studies conducted by Jagtap and Kleer et al. ${ }^{1,10}$. However the division into typical and atypical MBC has prognostic significance ${ }^{1,16}$

\section{Conclusion}

$\mathrm{MBC}$ is a unique type of breast carcinoma with good prognosis. Larger studies are essential to understand the tumour biology in MBC. Atypical MBC have histological features of IDC as well. Histopathology plays an important role in diagnosing variants of $\mathrm{MBC}$ since the prognosis varies.

\section{Reference}

1. Jagtap VS, Beniwal A, Shah HP et al. Medullary carcinoma of breast :Clinicopathological study. J evid based med health 2016; 3(45):2256-60

2. Chu Z, Lin H, Liang X, Huang R, Zhan Q, Jiang J et al. Clinicopathological characteristics of Typical medullary breast carcinoma: A retrospective study of 117 cases.PLoS ONE 2014;9(11): E111493.

3. Juan R, Breast In Rosai and Ackerman's surgical pathology. $10^{\text {th }}$ edition, Elsiever,Mosby,2011:1660-1773.
4. Tavasolli FA, Devilee P. World health organization classification of tumors,Pathology and genetics of tumors of breast and female genital organs. Lyon: IARC press;2003.

5. Marginean F, Rakha AE, Ho BC, Ellis IO, Lee AH. Histological features of medullary carcinoma and prognosis in triple negative basal like carcinomas of the breast. Modern pathology 2010;23:1357-63.

6. Sloane JP. Biopsy pathology of the breast. $2^{\text {nd }}$ edition. New York : Oxford University Press;2001.

7. Moore OS, Foote FW. The relatively favourable prognosis of medullary carcinoma of the breast. Cancer 1949;2(4):635-64.

8. Chetty R. Gastrointestinal cancers accompanied by a dense lymphoid component: an overview with special reference to gastric and colonic medullary and lymphoepithelioma-like carcinomas. ClinPathol 2012;65:1062-1065.

9. Ridolfi RL, Rosen PP, Port A, et al. Medullary carcinoma of the breast: a clinicopathological study with 10 year followup. Cancer 1977;40(4):1365-1385.

10. Kleer CG. Carcinoma of the breast with medullary-like features Diagnostic challenges and relationship with BRCA1 and ERH2 functions. Arch Pathol Lab med 2009;133:1822-1825.

11. Rosen PP, Lesser ML, Kinne DW, et al. Discontinuous or "skip" metastases in breast carcinoma. Ann Surg 1983; 197:276-283

12. DabbsDJ, Specailtypes ofinvasive breastcarcinoma. In Breast pathology. $2^{\text {nd }}$ edition, Elseiver, Philadelphia,2017:639-662.

13. Santosh T, Bal AK, Patro MK, Choudhury A. Medullary Breast Carcinoma: A Case Report and Review of Literature. J Cancer SciTher 2015;7: 142-144.

14. Rosen PP. Medullary Carcinoma. In Rosen ${ }^{\mathrm{TM}}$ Breast Pathology. $3^{\text {rd }}$ edition, Lippincott Williams \& Wilkins, Philadelphia, 2009: 449-466.

15. Mateo AM, Pezzi TA, Sundermeyer M, Kelly C, Klimberg VS, Pezzi CM. Atypical medullary carcinoma of the breast has similar prognostic factors and survival to typical medullary carcinoma:3976 cases from the national cancer data base. J SurgOncol 2016;114(5):533-536.

16. Patil SM, Bhatia A, Karthik K, Umeshchandra DG. Medullary carcinoma breast: A case report. Sch. J. App. Med. Sci., 2013; 1(6):681-683

*Corresponding author:

Dr B R Vani, ESI Medical college \& Post Graduate Institute of Medical Science and Research, Rajajinagar, Bangalore (India) Phone: +91 8023325130

Email: vanibr@yahoo.in 